\title{
Potential role of coronary vasoconstriction in ischaemic heart disease: effect of exercise
}

\author{
${ }^{*}$ O. M. Hess, M. Būchi*, R. Kirkeeide ${ }^{* *}$, P. Niederer $\dagger$, M. Anliker $\dagger$, K. L. Gould** \\ AND H. P. KRAYENBÜHL* \\ * Department of Internal Medicine, Medical Policlinic, Cardiology, University Hospital and \\ † Institute of Biomedical Engineering, University of Zürich, ** Division of Cardiology, \\ University of Texas, Houston, U.S.A.
}

KEY WORDS: Vasoconstriction, coronary circulation, myocardial ischaemia, exercise.

Coronary vasomotion plays an important role in the regulation of coronary perfusion at rest and during exercise. Normal coronary arteries show coronary vasodilation of the proximal $(+20 \%)$ and distal $(+40 \%)$ vessel segments during supine bicycle exercise. However, patients with coronary artery disease show exercise-induced vasoconstriction of the stenotic vessel segments. The exact mechanism of exercise-induced stenosis narrowing is not clear but might be related to a passive collapse of the disease-free vessel wall (Venturi mechanism), elevated plasma levels of circulating catecholamines, an insufficient production of the endothelium-derived vasorelaxing factor or increased platelet aggregation due to turbulent blood flow with release of thromboxane $A_{2}$ and serotonin.

Various vasoactive drugs, such as nitroglycerin and calcium antagonists, prevent exercise-induced stenosis vasoconstriction. An additive effect on coronary vasodilation of the stenotic vessel segment was observed after combination of nitroglycerin with diltiazem. Thus, exercise-induced stenosis narrowing plays an important role in the pathophysiology of myocardial ischaemia during dynamic exercise. The antianginal effect of vasoactive substances can be explained-besides the effect on preand afterload - by a direct action on coronary stenosis vasomotion.

\section{Introduction}

Coronary vasomotion plays an important role in the pathophysiology of myocardial ischaemia. Stenotic coronary arteries show coronary vasomotion at the site of the stenosis ${ }^{|1-5|}$ since approximately $70 \%$ of all stenoses have a normal musculo-elastic wall segment ${ }^{[6,7]}$. Coronary vasoconstriction of the normal and the stenotic vessel segment has been reported during isometric handgrip exercise $\mathrm{e}^{[8]}$. This vasoconstriction is probably due to reflex activation of the sympathetic nervous system with increased levels of circulating catecholamines. The purpose of the present study is, therefore, to discuss the effect of dynamic exercise on coronary vasomotion in patients with coronary artery disease and to evaluate the effect of various

Address for correspondence: Otto M. Hess M.D., Medical Policlinic, Cardiology, University Hospital, Raemistrasse 100, 8091 Zürich, Switzerland. vasoactive drugs on coronary vasomotion at rest and during exercise.

\section{Exercise protocol}

Simultaneous biplane coronary arteriography was used for quantitative evaluation of normal and stenotic coronary vessel segments at rest and during supine bicycle exercise. Patients were selected on a consecutive basis when a history of stable, effort-related angina pectoris and a clearly visible coronary stenosis were present. Quantitative biplane analysis was performed in the right and left anterior oblique projection but in some patients cranio-caudal angulation was necessary for proper visualization of the stenotic segment. Cinefilm was used as a data carrier (filming rate 50 frames $\mathrm{s}^{-1}$ ). Two different contrast materials were used: Before January $19885-8 \mathrm{ml}$ of an ionic contrast material (amidotrizoate; Urografin-76\%) was used for coronary arteriography ${ }^{[1.2 .5]}$. After January 1988 $5-8 \mathrm{ml}$ of a nonionic contrast material 
(iopamidol-trometamol; lopamiro 370) was used for coronary arteriography to minimize hyperaemic reactions of the coronary vessels. However, repeated injections with the ionic contrast material, amidotrizoate, produced only minor changes in coronary artery dimensions when these measurements were carried out at the end of the diagnostic coronary arteriography ${ }^{\mid 2]}$. Control biplane arteriography was performed with the patient's feet in elevated position attached to the bicycle ergometer. Exercise was begun at a level of 50-75 W which was increased every $2 \mathrm{~min}$ in increments of $25-50 \mathrm{~W}$. Biplane coronary arteriograms with concurrent aortic and pulmonary artery pressure recordings were obtained every $2 \mathrm{~min}$ and at the end of exercise. Sublingual nitroglycerin $(1.6 \mathrm{mg})$ was administered immediately at the end of the exercise test and repeat coronary arteriography was carried out $5 \mathrm{~min}$ thereafter.

\section{Quantitative coronary arteriography}

Quantitative evaluation of biplane coronary arteriograms was performed with two different systems: Before November $1988^{\{1,2,4,5\}}$ the outlines of coronary arteries were traced manually (4 to 6 times) in a blinded fashion and were digitized with an electronic digitizer (Numonics Corp.) interfaced to a computer PDP $11 / 34$. A portion of the catheter of known dimension was traced for each cineframe and was used as a scaling factor. Interobserver variability for this manual system was found to be small with a standard error of estimate (SEE) for biplane data of $0.30 \mathrm{~mm}^{2}(=7.9 \%$ of the mean vessel area).

After November 1988 an automatic system was used for quantitative coronary arteriography $^{(9)}$. The system is based on a $35 \mathrm{~mm}$ film projector, a slow-scan CCD-camera (image digitation) and a computer work-station (Apollo DN 3000; image storage and processing). Calibration is performed by the isocentre technique, which is based on two fixed reference points in the centre of the two image intensifiers. Contour detection is carried out in biplane projection using a geometric-densitometric edgedetection algorithm (Fig. 1). Interobserver variability was found to be small with a standard error of estimate for biplane data of $0.14 \mathrm{~mm}^{2}$ $(=4.1 \%$ of the mean vessel area).
The methodology for computerized analysis of coronary arteriograms has been described elsewhere ${ }^{[10]}$. Briefly, a three-dimensional model of the vessel is constructed by matching centre lines of the individual biplane tracings, assuming the vessel cross-section to be ellipsoidal. The proximal and distal as well as the minimal cross-sectional areas of the vessel segment are calculated and printed out by the computer.

\section{Normal coronary vasomotion}

Coronary vasomotion was studied at rest and during bicycle exercise in seven normal patients with no evidence of coronary artery disease and normal coronary flow reserve as assessed by coronary sinus thermodilution before and after intravenous dipyridamole ${ }^{|4|}$. Coronary luminal area of the left anterior descending or the left circumflex coronary artery were analysed at rest, during exercise and after $1.6 \mathrm{mg}$ nitroglycerin administered at the end of the exercise test. Coronary luminal area increased by $20 \%$ during exercise and by $45 \%$ after sublingual administration of nitroglycerin. Thus, in normal patients coronary vasodilation occurs during dynamic exercise which is, however, not maximal, since after administration of nitroglycerin coronary luminal area is approximately double that during exercise.

\section{Coronary artery disease}

Coronary vasomotion of stenotic coronary arteries represents a significant element in the pathogenesis of myocardial ischaemia ${ }^{[7]}$. Brown and coworkers ${ }^{[8 \mid}$ have reported a decrease in coronary luminal areas of normal and stenotic vessel segments by $14 \%$ and $35 \%$, respectively, during isometric handgrip exercise. Coronary vasoconstriction was prevented after intracoronary infusion of nitroglycerin during a second handgrip test. Thus, active coronary vasoconstriction during handgrip exercise is probably due to reflex activation of the sympathetic nervous system and can be suppressed by the use of intracoronary nitroglycerin. Gage et al. ${ }^{[1]}$ and Gordon et al. ${ }^{|3|}$ reported a decrease in coronary luminal area of stenotic coronary arteries during dynamic exercise, whereas normal vessel segments showed exercise-induced vasodilation. This exercise-induced stenosis narrowing is dependent on the severity of coronary stenosis $^{(3)}$, since mild lesions show only 
Figure 1 Quantitative evaluation of a coronary arteriogram in a patient with a severe stenosis of the left circumflex coronary artery. The arteriogram is projected on a $35 \mathrm{~mm}$ film projector, digitized on a slow-scan CCD-camera and processed on an Apollo DN 3000 computer work-station. Contour detection is performed automatically in biplane projection using a geometric-densitometric edge-detection algorithm. Luminal areas of the normal and stenotic vessel segment are given in both projections as well as in biplane projection (top left panel). The patient's data are included in the right hand panel.

minimal $(-10 \%$ area reduction, NS vs rest) but severe lesions significant $(-32 \%$ area reduction, $P<0.001$ vs rest) vasoconstriction during bicycle exercise. The exact mechanism of this exerciseinduced stenosis narrowing is not clear but might involve the following: (1) passive collapse of the disease-free vessel wall within the stenosis due to an increase in blood flow velocity during exercise (=Venturi mechanism); (2) active vasoconstriction due to increased levels of circulating catecholamines during exercise; (3) insufficient production of the endotheliumderived vasorelaxing factor during exercise due to the atherosclerotic alterations of the vessel wall ${ }^{[1]} ;(4)$ increased platelet aggregation during exercise due to turbulent blood flow behind the stenosis with release of thromboxane $A_{2}$ and serotonin $^{[2]}$. It is likely that more than one of these mechanisms combine to produce the observed stenosis vasoconstriction during bicycle exercise. To examine further this phenomenon we studied the effect of different vasoactive substances on coronary vasomotion of the stenotic vessel segment.

\section{Effect of vasoactive drugs on coronary vasomotion}

The effect of intracoronary nitroglycerin on coronary vasomotion of normal and stenotic vessel segments (1) was evaluated in six patients with coronary artery disease at rest and during supine bicycle exercise. Immediately prior to the exercise test, $0 \cdot 1 \mathrm{mg}$ intracoronary nitroglycerin was administered which was followed by two levels of bicycle exercise. A normal (Fig. 3) and a stenotic (Fig. 4) vessel segment were evaluated quantitatively and compared with a control group of 12 patients with coronary artery disease but without intracoronary nitroglycerin before the exercise test ${ }^{\prime \prime}$. Immediately at the end of the exercise test $1.6 \mathrm{mg}$ sublingual nitroglycerin was administered to both groups.

The normal vessel segment (Fig. 3) showed 

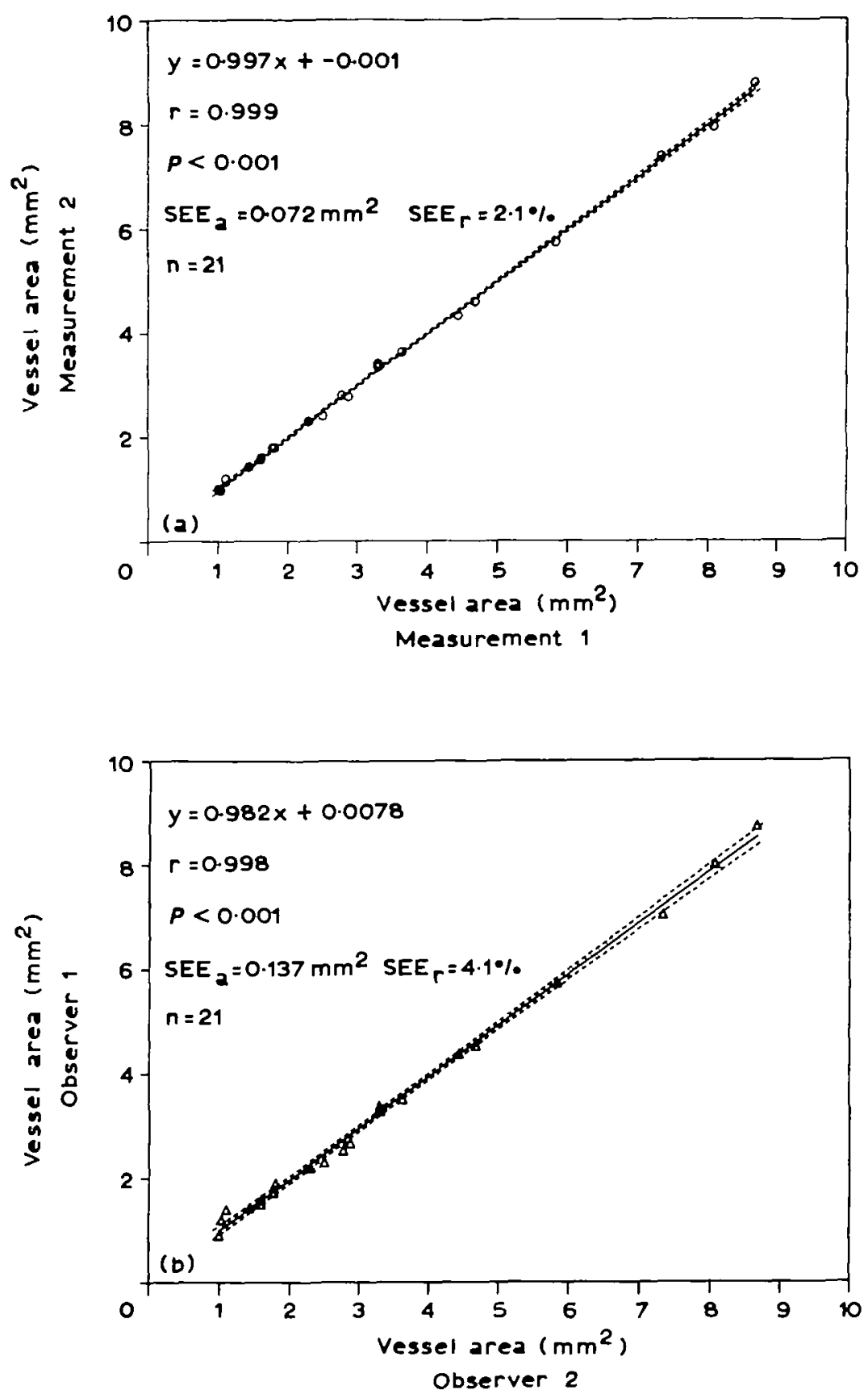

Figure 2 Intraobserver (a) and interobserver (b) variability for quantitative coronary arteriography using an automatic contour detection algorithm. The standard error of estimate (SEE) was $0.076 \mathrm{~mm}^{2}$ or $2.1 \%$ of the mean (biplane) vessel area (intraobserver variability) and was $0.137 \mathrm{~mm}^{2}$ or $4.1 \%$ of the mean (biplane) vessel area (interobserver variability). With the use of an automatic system, the standard error (interobserver variability) was reduced from $7.9 \%$ (manual system) to $4.1 \%$ (automatic system). $r$ : correlation coefficient, $P$ : probability, $n$ : number of measurements. 


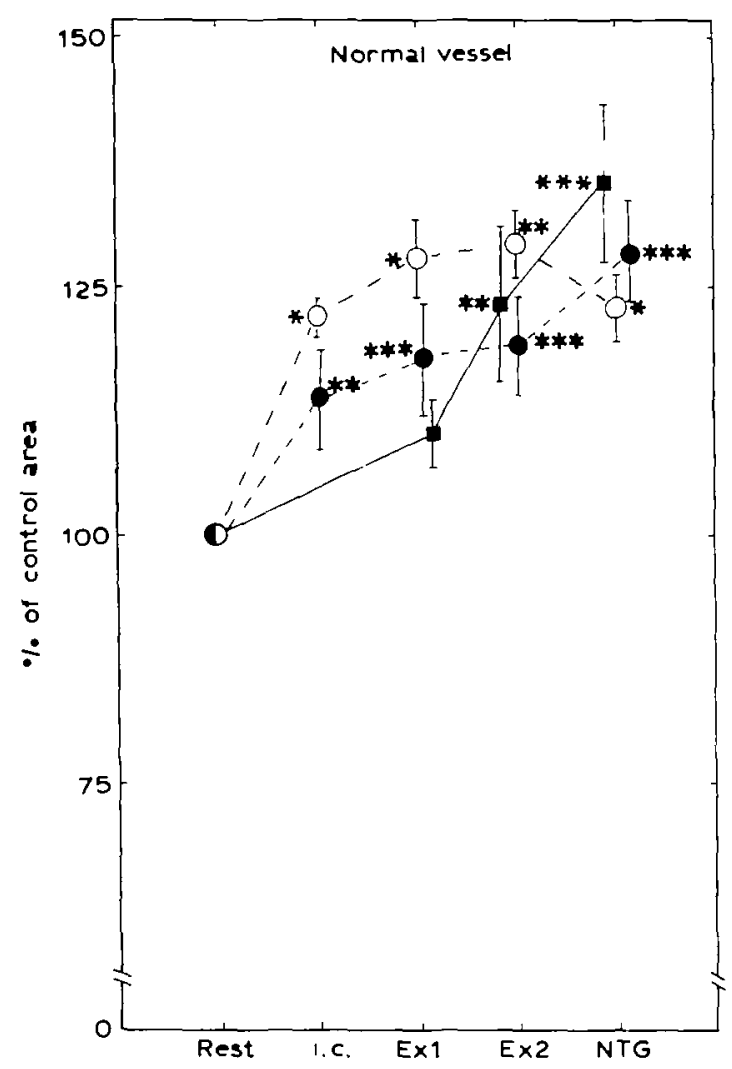

Figure 3 \% change of the normal vessel area during bicycle exercise (Ex1 and Ex2) and after sublingual administration of $1.6 \mathrm{mg}$ nitroglycerin (NTG). Three groups of patients were studied: group 1 served as control group and received no pretreatment prior to the exercise test, group 2 received $0 \cdot 1 \mathrm{mg}$ intracoronary (i.c.) nitroglycerin prior to exercise and group $32-3 \mathrm{mg}$ intracoronary diltiazem. The control group showed coronary vasodilation during submaximal exercise $(E x 2)$, whereas in the other two groups vasodilation was observed after intracoronary injection of the drug; nitroglycerin showed a more pronounced vasodilator effect on the normal coronary arteries than diltiazem (for explanations see text). $\mapsto-1=$ mean \pm 1 standard error; * $=P<0.05, \quad{ }^{*}=P<0.01, * *=P<0.001$ vs rest. $\square=$ control; $\mathrm{O}-\mathrm{O}=\mathrm{NTG} ; \mathrm{O} \cdot-\mathrm{O}=$ diltiazem.

coronary vasodilation after intracoronary injection of nitroglycerin $(+21 \%, P<0.05$ vs rest) which persisted during maximum exercise $(+29 \%, P<0.01$ vs rest) and after sublingual nitroglycerin $(+23 \%, P<0.05$ vs rest). The stenotic vessel segment (Fig. 4) also showed coronary vasodilation after intracoronary administration of nitroglycerin $(+22 \%, P<0.05$ vs

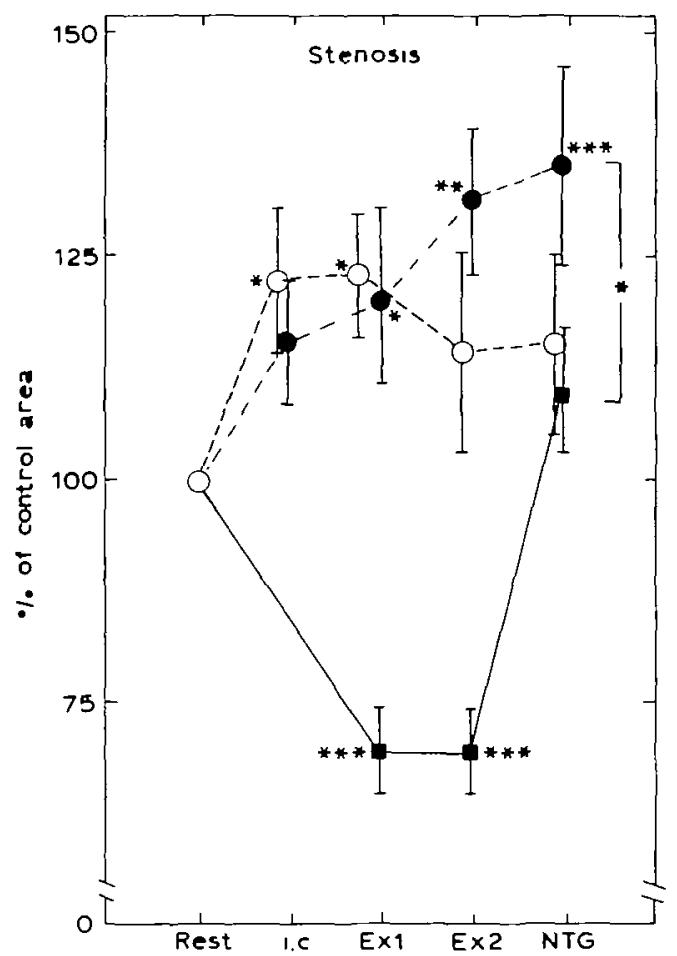

Figure $4 \%$ change of the stenotic vessel area during bicycle exercise (Ex1 and Ex2) and after sublingual administration of $1.6 \mathrm{mg}$ nitroglycerin (NTG). The same three groups as shown in Fig. 3 were studied: group 1 served as control group without intracoronary pretreatment prior to the exercise test, group 2 received $0.1 \mathrm{mg}$ intracoronary (i.c.) nitroglycerin prior to exercise and group $32-3 \mathrm{mg}$ intracoronary diltiazem. In contrast to the normal vessel segment, there is exercise-induced stenosis narrowing in the control group which is, however, reversible after sublingual administration of nitroglycerin. Intracoronary administration of nitroglycerin or diltiazem prevented stenosis narrowing during exercise when the drug was injected prior to the exercise test. The combination of intracoronary diltiazem with sublingual nitroglycerin showed an additive effect which was significantly $(P<0.05)$ more pronouned after this combination than after sublingual nitroglycerin alone (for further explanations sec text). Abbreviations and symbols are as in Fig. 3.

rest) which persisted during the first 2 min of exercise $(+23 \%, P<0.05$ vs rest) but decreased slightly towards resting levels during maximum exercise $(+14 \%$, NS vs rest) and after sublingual administration of nitroglycerin $(+16 \%$, NS vs rest).

Thus, intracoronary administration of nitroglycerin is associated with an increased 
coronary luminal area in normal and stenotic vessel segments. During bicycle exercise no further dilation of the coronary arteries was observed probably owing to maximal predilation of the coronary arteries after intracoronary nitroglycerin. Thus, exercise-induced stenosis narrowing is eliminated (Fig. 4) due to the strong vasodilating effect of nitroglycerin on the smooth vasculature.

The effect of $2-3 \mathrm{mg}$ intracoronary diltiazem on coronary vasomotion of normal and stenotic vessel segments was evaluated in a third group of nine patients with coronary artery disease ${ }^{[13]}$ and compared with the data from 12 patients with coronary artery disease not given pretreatment prior to the exercise test ${ }^{[1]}$.

Intracoronary administration of diltiazem was associated with coronary vasodilation of the normal $(+14 \%, P<0.01$ vs rest) and stenotic $(+15 \%$, NS vs rest) vessel segment which was, however, slightly less than after intracoronary nitroglycerin (Figs 3 and 4). During submaximal bicycle exercise (EX2), luminal area of both normal $(+19 \%, P<0.001$ vs rest $)$ and stenotic ( $+31 \%, \quad P<0.01$ vs rest) vessel segments further increased. After sublingual administration of nitroglycerin in the patients given intracoronary diltiazem, there was an additive effect on coronary vasodilation of the stenotic vessel segment $(+35 \%, P<0.001$ vs rest); the $\%$ increase in luminal area was significantly $(P<0.05)$ more pronounced after diltiazem and nitroglycerin than after sublingual nitroglycerin alone (Fig. 4).

Thus, diltiazem exhibits a vasodilatory effect on both normal and stenotic vessel segments which is less pronounced than that after intracoronary nitroglycerin (Figs 3 and 4). Similar observations have been made in the chronically instrumented $\operatorname{dog}^{[14]}$; it was shown that the calcium antagonists nifedipine, verapamil and diltiazem are relatively weak coronary vasodilators compared with nitroglycerin. The reason nitroglycerin appears to be a more potent dilator of epicardial coronary arteries than calcium antagonists is unclear ${ }^{(14)}$. The additive effect of diltiazem and nitroglycerin was observed in the present study only in stenotic (Fig. 4) but not in normal (Fig. 3) vessel segments. The mechanism of this additive effect is not clear but might be related to the different mode of action of the two vasodilators: diltiazem reduces the calcium influx into the smooth muscle cell; nitroglycerin increases the cyclic guanosine monophosphate in the vascular musculature. Apparently, the combined action of these two vasodilators enhances the vasodilating properties of each drug alone. Both drugs are well established in the treatment of patients with coronary artery disease and both have been shown to be highly efficient in reducing myocardial ischaemia ${ }^{15,16]}$. Calcium antagonists and nitrates are often combined in the daily routine for an optimal antiischaemic effect in patients with angina pectoris although clinical trials have not shown a better result in terms of anginal pain and physical working capacity for the combined regimen than for either drug alone ${ }^{[16]}$.

\section{References}

[1] Gage JE, Hess OM, Murakami T, Ritter M, Grimm J, Krayenbuehl HP. Vasoconstriction of stenotic coronary arteries during dynamic exercise in patients with classic angina pectoris: reversibility by nitroglycerin. Circulation 1986; 73: 865-76.

[2] Gaglione A, Hess OM, Corin WJ, Ritter M, Grimm J, Krayenbuehl HP. Is there coronary vasoconstriction after intracoronary beta-adrenergic blockade in patients with coronary artery disease? J Am Coll Cardiol 1987; 10: 299-310.

[3] Gordon JB, Zebede J, Wayne RR, Mudge GH, Ganz P, Selwyn AP. Coronary constriction with exercise: possible role for endothelial dysfunction and alpha tone. Circulation 1986; 74 (Suppl II): II 481 (Abstr)

[4] Bortone AS, Hess OM, Eberli FR et al. Abnormal coronary vasomotion during exercise in patients with normal coronary arteries and reduced coronary flow reserve. Circulation 1989; 79: 516-27.

[5] Hess OM, Bortone AS, Eid $\mathbf{K}$ et al. Coronary vasomotor tone during static and dynamic exercise. Eur Heart J 1989; 10: 105-10.

[6] Freudenberg $H^{-}$, Lichtlen PR. The normal wall segment in coronary stenosis - a postmortem study. Z Kardiol 1981; 70: 863-9.

[7] Brown BG, Bolson EL, Dodge HT. Dynamic mechanisms in human coronary stenosis. Circulation $1984 ; 42$ : 917-22.

[8] Brown BG, Lee AB, Bolson EL, Dodge HT. Reflex constriction of significant coronary stenosis as a mechanism contributing to ischemic left ventricular dysfunction during isometric exercise. Circulation 1984; 70: 18-24.

[9] Büchi M, Hess OM, Suter Thet al. Validation of a new automatic system for biplane quantitative coronary arteriography. Int J Cardiovase Imaging, in press.

[10] Kirkeeide RL, Gould KL. Cardiovascular imaging: Coronary artery stenosis. Hosp Pract 1984; 19: 160-3.

[11] Bassenge $E$, Stewart DJ. Interdependence of pharmacologically induced and endothelium- 
mediated coronary vasodilation in antianginal therapy. Cardiovasc Drugs Ther 1988; 1: 47-55.

[12] Folts JD, Gallagher $K$, Rowe GG. Blood flow reductions in stenosed canine coronary arteries: vasospasm or platelet aggregation? Circulation 1982; 65: 248-55.

[13] Nonogi H, Hess OM, Ritter $M$ et al. Prevention of coronary vasoconstriction by diltiazem during dynamic exercise in patients with coronary artery disease. J Am Coll Cardiol 1988; 12: 892-9.

[14] Schwartz JS, Bache RJ. Combined effects of calcium antagonists and nitroglycerin on large coronary artery diameter. Am Heart J 1988; 115: 964-9.

[15] Boden WE, Bough EW, Reichmann MJ et al. Beneficial effects of high-dose diltiazem in patients with persistent effort angina on beta-blockers and nitrates: a randomized, double-blind placebocontrolled cross-over study. Circulation 1985; 71: 1197-205.

[16] Hossack KF, Eldridge JE, Buckner K. Comparison of acute hemodynamic effects of nitroglycerin versus diltiazem and combined acute effects of both drugs in angina pectoris. Am J Cardiol 1986; 58: 722-6. 